\title{
Fullerenes finally score as Nobel committee honours chemists
}

London. The Nobel committee last week gave the answer to a favourite topic of speculation at chemistry conferences for several years: when would the chemistry prize be awarded for the discovery that carbon atoms can assemble into the $\mathrm{C}_{60}$ carbon cages of buckminsterfullerene - and who would win it?

Both questions have now been answered, the second with the decision to award the prize to Sir Harry Kroto of the UK University of Sussex (bottom right), and Robert Curl and Richard Smalley of Rice University in Houston, Texas (right).

The discovery of buckminsterfullerene, named after the American architect Buckminster Fuller, who used similar geodesic domes to construct large-scale structures, was made at Rice University in 1985 during experiments to investigate carbon clusters formed by laser ablation of graphite.

That the discovery of $\mathrm{C}_{60}$ would one day earn Nobel recognition has been widely regarded as inevitable. It is hard to identify any other recent finding in chemistry that has stimulated such intense and diverse activity throughout the physical sciences.

Organic chemists have literally found a new dimension for synthesis in $\mathrm{C}_{60}$ 's spherical topology. Solid-state physicists have scaled new heights in molecular superconductivity. Films of solid $\mathrm{C}_{60}$ doped with alkali metals are superconducting at up to 33 degrees kelvin, $20 \mathrm{~K}$ above the previous record for a molecular superconductor.

$\mathrm{C}_{60}$ exhibits novel tribological effects and promising nonlinear optical properties, and is a candidate for the carrier of unexplained features in astronomical spectra. "It has completely changed our perspective on carbon chemistry," says Kroto.

The question was never so much whether $\mathrm{C}_{60}$ would win a Nobel prize as when, and most of all - for whom. David Jones, a chemist at the University of Nottingham, says he had expected that the award might be given "only when $\mathrm{C}_{60}$ turned out to be of some use".

But commercial applications remain remote, and Jones considers that $\mathrm{C}_{60}$ and the related carbon-cage clusters collectively called fullerenes have so far done little of practical value "other than providing entertainment". (Jones's speculations about a curved form of graphite in his Daedalus column in New Scientist in 1966 are commonly regarded as the conceptual beginning of fullerene science.)

In contrast, Robert Haddon, one of the team at Bell Laboratories in New Jersey who discovered superconducting $\mathrm{C}_{60}$ compounds in 1991, feels that the prize "could have been awarded by the end of 1991, when it was clear that fullerenes would change organic chemistry and materials science".

The question of 'who' was the hardest, as the route from discovery to worldwide impact has involved many significant contributions. But no award would have made sense that omitted Kroto, Smalley or Curl.

\section{IMAGE UNAVAILABLE FOR COPYRIGHT REASONS}

\begin{abstract}
Robert Curl (above left), his colleague Richard Smalley (on screen behind model), and Harry Kroto (below right) featured on Nature's front page (left) in 1985 with their report of a discovery that has transformed knowledge of nanoscale carbon structures.
\end{abstract}

Kroto's interest in long-chain carbon molecules, called polyynes, as the source of features in the microwave spectra of interstellar clouds stimulated the experiments at Rice, where Curl introduced Kroto to the laser-ablation apparatus that he and Smalley were using to study semiconductor clusters.

The experiments on graphite were initiated to look at clusters of up to around 33 atoms, which were expected to be linear chain molecules. $\mathrm{C}_{60}$ and the other cage-like fullerenes were an unexpected by-product. The peculiar stability of $\mathrm{C}_{60}$ posed a puzzle that demanded explanation.

But perfecting the conditions required for optimal $\mathrm{C}_{60}$ formation was a task performed by graduate students Jim Heath and Sean O'Brien (and also initially Yuan Liu and Qing-ling Zhang) at Rice University. Heath and O'Brien co-authored with Kroto, Curl and Smalley the 1985 paper that reported the discovery (see Nature 318, 162: 1985).
$\mathrm{C}_{60}$ might well have stayed the curiosity it remained for the next five years if physicists Wolfgang Kratschmer, Don Huffman and their respective students Kosta Fostiropoulos and Lowell Lamb had not found a way to make the compound in gram quantities in 1990. It was this discovery that allowed fullerene science to blossom.

There is no question that fullerenes have provided entertainment for scientists and non-scientists alike. But how important are they? Kroto feels that the Nobel committee have taken "a gamble that fullerenes will be one of the biggest things in the 21st cen당 tury". In Haddon's view, the significance 韸 is in fundamental, not applied, science. के "It's the kind of fundamental breakthrough in chemistry of the kind we don't really see any more," he says.

Curl is confident that technological uses will come in the next few decades. But he suggests that the most telling factor now is that fullerenes illustrate "the interplay of [molecular] topology and structure". Fullerene science has led to the discovery of a host of materials, carbon-based and otherwise, whose atomicscale structure dictates particular nanoscale shapes and forms.

Carbon nanotubes, discovered in 1991, are the most celebrated example: tubes of graphite-like carbon a few nanometres in diameter whose closed end caps are curved by the same five-membered-ring defects that induce the curvature of the $\mathrm{C}_{60}$ cage.

Smalley agrees that "the utility of $\mathrm{C}_{60}$ is in what it has taught us about nanoscale carbon structures" - namely that the graphite sheet "is a pretty cool thing". Fullerenes may turn out to be not so much an end in themselves as the beginning of a new view of materials science at the interface of the molecular and the bulk scales.

Philip Ball

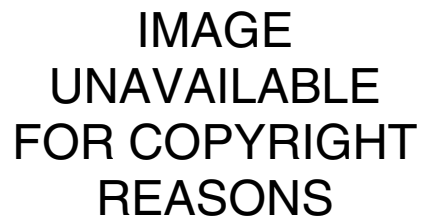

IMAGE REASONS 